PROCEEDINGS OF THE

AMERICAN MATHEMATICAL SOCIETY

Volume 140, Number 3, March 2012, Pages 855-863

S 0002-9939(2011)11125-0

Article electronically published on June 29, 2011

\title{
EULER OBSTRUCTION AND POLAR MULTIPLICITIES OF IMAGES OF FINITE MORPHISMS ON ICIS
}

\author{
R. CALLEJAS-BEDREGAL, M. J. SAIA, AND J. N. TOMAZELLA
}

(Communicated by Ted Chinburg)

\begin{abstract}
We show how to compute the local polar multiplicities of a germ at zero of an analytic variety $Y$ in $\mathbb{C}^{p}$, which is the image by a finite morphism $f: Z \rightarrow Y$, of a $d$-dimensional isolated complete intersection singularity $Z$ in $\mathbb{C}^{n}$. We also show how to compute the local Euler obstruction of $Y$ at zero in the case that it is reduced. For this we apply the formula due to Lê and Teissier which describes the local Euler obstruction as an alternating sum of the local polar multiplicities.
\end{abstract}

\section{INTRODUCTION}

The computation of numerical invariants associated to a germ of a complex analytic set is very important to describe geometrical and topological properties of such a germ. For instance, the local Euler obstruction was one of the main tools defined by MacPherson to prove the conjecture of Deligne and Grothendieck on the existence of Chern classes for singular complex algebraic varieties. With the aid of the purely algebraic interpretation of the local Euler obstruction given by Gonzalez-Sprinberg, Lê and Teissier in $[8$ gave a description of the local Euler obstruction as an alternating sum of the multiplicities of the local polar varieties of such a germ. These polar multiplicities are also very important for studying the geometry of any germ of an analytic complex variety and there are several works showing it. Teissier in 14 gives an algorithm for obtaining the canonical Whitney stratification of complex sets in terms of the polar multiplicities. They are also the main invariants to guarantee the Whitney equisingularity of families of germs of analytic complex varieties (see [14]) and of one-parameter families of finitely determined map germs (see [1]).

However it is not easy to compute these invariants if we do not know the geometrical structure of the germ. Gaffney in [1] shows how to compute the polar multiplicities of a very special class of germs of complex sets: the stable singularities which appear in the discriminant of any co-rank one finitely determined map germ from $\mathbb{C}^{2}$ to $\mathbb{C}^{2}$ or from $\mathbb{C}^{2}$ to $\mathbb{C}^{3}$. Since there is no direct way to compute

Received by the editors April 6, 2009 and, in revised form, December 7, 2010.

2010 Mathematics Subject Classification. Primary 32S30; Secondary 32S10.

Key words and phrases. Local Euler obstruction, polar multiplicity, complete intersection.

The first-named author is partially supported by CAPES-Procad grant 190-2007; CNPq, grant 620108/2008-8; and FAPESP, grant 2010/03525-9.

The second- and third-named authors are partially supported by CNPq, grant 300733/2009-7; CAPES, grant 222/2010; and FAPESP, grant 2008/54222-6.

(C)2011 American Mathematical Society Reverts to public domain 28 years from publication 
these invariants, Gaffney shows how to compute them in terms of Milnor numbers of isolated complete intersection singularities (ICIS for short) that are in the critical set of the germ in the source; for this he strongly uses the fact that the map germ is finite when restricted to these sets. More recently this method was applied to the computation of the polar multiplicities of the stable singularities which appear in the discriminant of any co-rank one finitely determined map germ from $\mathbb{C}^{n}$ to $\mathbb{C}^{p}$, in [4] for $n<p$ and in [5] for $n=p$, with the pair $(n, p)$ in the nice dimensions of Mather or in its boundary.

In this article we apply this method to compute the local polar multiplicities at zero of any reduced complex analytic set $Y^{d}$ in $\mathbb{C}^{p}$, which is the image by a finite morphism, of a germ at zero of a $d$-dimensional ICIS $Z^{d}$ in $\mathbb{C}^{n}$. We show how to compute the local polar multiplicities of $Y^{d}$ in terms of the Milnor numbers of the ICIS $Z^{d}$ and other ICIS which lie in $Z^{d}$, generalizing the results above.

As an application we show how to compute the local Euler obstruction of $Y^{d}$ at zero. In order to show this result we apply the description of the local Euler obstruction given by Lê and Teissier as an alternating sum of the local polar multiplicities.

To conclude, we compare our results with some special cases that recently appeared in the literature.

\section{Finite MORPhisms AND DEGREE}

The co-length of a given ideal $I$ in a complex analytic ring $R$ is defined as $\operatorname{dim}_{\mathbb{C}}(R / I)$. The Hilbert-Samuel multiplicity of an ideal $I$ is an integer denoted by $e(I)$ that is defined whenever $I$ has finite co-length. We remark that along this paper we shall compute the multiplicity for $\mathfrak{m}$-primary complete intersection ideals $I$ in rings $R=(R, \mathfrak{m})$ which are local and Cohen-Macaulay. In this case the multiplicity of $I$ is just its co-length.

A smooth map germ $f:(Z, x) \rightarrow(Y, y)$ is said to be finite if the dimension of its local algebra is finite, i.e. if the number $m(f):=\operatorname{dim}_{\mathbb{C}} \frac{\mathcal{O}_{(Z, x)}}{f^{*}\left(\mathfrak{m}_{y}\right) \mathcal{O}_{(Z, x)}}$ is finite. Note that for $f$ to be finite, it is necessary that $\operatorname{dim} Z \leq \operatorname{dim} Y$. In the context of complex analytic geometry we have the following important result for finite maps; see [12, section 3.12] for more details.

Let $(Z, x)$ and $(Y, y)$ be germs of analytic spaces of the same dimension and $f:(Z, x) \rightarrow(Y, y)$ be an analytic map such that $f(Z)$ is Zariski-dense in $Y$. Suppose $f(x)$ is a smooth point of $Y$, and $\{x\}$ a component of the fiber $f^{-1}(f(x))$. For open neighborhoods $U \subset Z$ of $x, V \subset Y$ and a closed analytic subset $B \subset Y$ such that:

(i) $V \backslash B$ is connected,

(ii) $f(U) \subset V,\left.f\right|_{U}$ is proper, $f^{-1}(f(x))=\{x\}$ and

(iii) $\left.f\right|_{U \backslash f^{-1}(B)}$ is smooth,

then the number of pre-images in $U$ of any point $y \in V \backslash B$, counted with multiplicity, is called the degree of $f$ at $x$, denoted by $\operatorname{deg}(f)$.

Remark 2.1. In particular if $(Z, 0) \subset\left(\mathbb{C}^{n}, 0\right)$ is an analytic space germ of dimension $d, \mathcal{O}_{Z}$ is Cohen-Macaulay and $\pi:\left(\mathbb{C}^{n}, 0\right) \rightarrow\left(\mathbb{C}^{d}, 0\right)$, defined as $\pi=\left(\pi_{1}, \ldots, \pi_{d}\right)$, is such that the restriction of $\pi$ to $Z$ is a finite map, then $\operatorname{deg}\left(\left.\pi\right|_{Z}\right)$ is the co-length of the ideal $\left(\pi_{1}, \ldots, \pi_{d}\right)$ in $\mathcal{O}_{Z}$, that is, $\operatorname{deg}\left(\left.\pi\right|_{Z}\right)=\operatorname{dim}_{\mathbb{C}} \frac{\mathcal{O}_{Z}}{\left(\pi_{1}, \ldots, \pi_{d}\right)}$. 


\section{Polar VARIETIES}

The polar varieties of analytic space germs were defined by Teissier in [14. We describe here this construction.

Suppose $(Z, 0) \subseteq\left(\mathbb{C}^{n}, 0\right)$ is a $d$-dimensional germ at zero of a complex analytic set. Let $p: \mathbb{C}^{n} \rightarrow \mathbb{C}^{d-k+1}$ be a generic linear projection such that $\operatorname{ker} p=D_{d-k+1}$ is a linear subspace of $\left(\mathbb{C}^{n}, 0\right)$ of dimension $n-(d-k+1)$, with $k=0, \ldots, d-1$. Denote by $p_{Z}: Z^{0} \rightarrow \mathbb{C}^{d-k+1}$ the restriction of $p$ to $Z^{0}:=Z-\operatorname{Sing}(Z)$, where $\operatorname{Sing}(Z)$ denotes the singular set of $Z$.

Definition 3.1. Let $P_{k}(Z, p)$ be the subspace of $Z$ defined as the closure of the points $x \in Z-\operatorname{Sing}(Z)$ such that $x$ is in the critical set $\Sigma\left(p_{Z}\right)$ of $p_{Z} . P_{k}(Z, p)$ is called the absolute polar variety associated to $Z$ with respect to $p$. We remark that as $p$ is generic, $P_{k}(Z, p)$ has codimension $k$ in $Z$.

The key invariant of $P_{k}(Z, p)$ is its multiplicity at zero, which we denote by $m_{0}\left(P_{k}(Z)\right)$ or $m_{k}(Z)$; it is called the $k^{\text {th }}$-polar multiplicity of $Z$ at 0 . We remember that for generic choice of $p$ this multiplicity is constant, hence an invariant of $Z$; therefore for any generic choice of linear projection $p$, we denote $P_{k}(Z, p)$ by $P_{k}(Z)$.

\section{Polar multiplicities of images of ICIS}

We fix the following setup: Let $Z$ be a germ at zero of an ICIS in $\mathbb{C}^{n}$ and $Y$ be a germ at zero of an analytic variety in $\mathbb{C}^{p}$, both of pure dimension $d$. Let $\bar{f}:\left(\mathbb{C}^{n}, 0\right) \rightarrow\left(\mathbb{C}^{p}, 0\right)$ be a finitely determined map germ such that $\bar{f}(Z, 0)=(Y, 0)$ and the restriction of $\bar{f}$ to $(Z, 0)$, denoted by $f:(Z, 0) \rightarrow(Y, 0)$, is finite. From now on, for any ideal $I$ generated by $\left\{g_{1}, \ldots, g_{r}\right\}$ we denote by $J(I)$ the ideal generated by the maximal minors of the Jacobian derivative matrix of $\left\{g_{1}, \ldots, g_{r}\right\}$. For any map germ $g$ we define the discriminant $\Delta(g)$ of $g$ by $\Delta(g)=g(\Sigma(g))$, where $\Sigma(g)$ is the critical locus of $g$.

For a fixed $k=0, \ldots, d-1$, choose a generic linear projection $p_{d-k+1}: \mathbb{C}^{p} \rightarrow$ $\mathbb{C}^{d-k+1}$ to obtain the polar variety $P_{k}(Y)=\overline{\Sigma\left(p_{d-k+1} \mid Y^{0}\right)}$.

To compute the multiplicities $m_{k}(Y)$, for $k=0, \ldots, d-1$, we need to consider the sets $\overline{\Sigma\left(p_{d-k+1} \mid Y^{0}\right)}$; however, these sets have the disadvantage that they are in the target and we do not know anything about their geometry which could help us to compute these multiplicities.

Using the fact that $f$ is finite we can work with the sets $Z_{k}=\overline{\Sigma\left(p_{d-k+1} \circ f \mid Z^{0}\right)}$, which are described as

$$
Z_{k}=V\left(\mathcal{I}(Z), J\left(p_{d-k+1} \circ f, \mathcal{I}(Z)\right)\right) .
$$

The advantage of working with these sets is that they are in the source and their equations are computable. Our strategy is to compute the polar multiplicities in the target in terms of some other invariants associated to these sets. First we show some preliminary results which are essential to prove our main results.

Lemma 4.1. For $k=1, \operatorname{deg}\left(p_{d-1} \circ f \mid Z_{1}\right)=\operatorname{deg}(f)\left[m_{1}(Y)+m_{0}(\Delta(f))\right]$.

For each $k=0, \ldots, d-1$, with $k \neq 1, \operatorname{deg}\left(p_{d-k} \circ f \mid Z_{k}\right)=\operatorname{deg}(f) m_{k}(Y)$.

Proof. Notice that if $Z_{k}=\overline{\Sigma\left(p_{d-k+1} \circ f \mid Z^{0}\right)}$, then $Z_{k}=f^{-1}\left(P_{k}(Y)\right) \cup \Sigma^{k}(f)$, where $\Sigma^{k}(f)$ denotes the Thom-Boardman space defined as

$$
\Sigma^{k}(f)=\overline{\left\{x \in X^{0}: \operatorname{rank}\left(d_{x}(f)\right) \leq d-k\right\}},
$$


which has dimension $d-k^{2}$. Hence

$$
\begin{aligned}
\operatorname{deg}\left(p_{d-k} \circ f \mid Z_{k}\right) & =\operatorname{deg}(f) \operatorname{deg}\left(p_{d-k} \mid f\left(Z_{k}\right)\right) \\
& =\operatorname{deg}(f) \operatorname{deg}\left(p_{d-k} \mid\left[P_{k}(Y) \cup f\left(\Sigma^{k}(f)\right)\right]\right. \\
& =\operatorname{deg}(f)\left[\operatorname{deg}\left(p_{d-k} \mid P_{k}(Y)+\operatorname{deg}\left(p_{d-k} \mid f\left(\Sigma^{k}(f)\right)\right)\right] .\right.
\end{aligned}
$$

Now since $p_{d-k}: \mathbb{C}^{d} \rightarrow \mathbb{C}^{d-k}$ is a generic linear projection and $P_{k}(Y)$ has dimension $d-k$, we obtain $\operatorname{deg}\left(p_{d-k} \mid P_{k}(Y)\right)=m_{k}(Y)$.

Moreover, as $\operatorname{dim}\left(\Sigma^{k}(f)\right)=d-k^{2}$ and $f$ is finite, the image $p_{d-k}\left(f\left(\Sigma^{k}(f)\right)\right)$ is nowhere dense in $\mathbb{C}^{d-k}$ if $k \neq 1$.

Therefore, for $k \neq 1$ we obtain $\operatorname{deg}\left(p_{d-k} \mid f\left(\Sigma^{k}(f)\right)\right)=0$ and

$$
\operatorname{deg}\left(p_{d-k} \circ f \mid Z_{k}\right)=\operatorname{deg}(f) m_{k}(Y) .
$$

On the other hand, $\Sigma^{1}(f)=\Sigma(f)$ is the critical locus of $f$; hence $f\left(\Sigma^{1}(f)\right)=$ $\Delta(f)$. Since $\operatorname{dim}(\Delta(f))=d-1$ we obtain

$$
\operatorname{deg}\left(p_{d-1} \mid f\left(\Sigma^{1}(f)\right)\right)=\operatorname{deg}\left(p_{d-1} \mid \Delta(f)\right)=m_{0}(\Delta(f)) .
$$

Therefore $\operatorname{deg}\left(p_{d-1} \circ f \mid Z_{1}\right)=\operatorname{deg}(f)\left[m_{1}(Y)+m_{0}(\Delta(f))\right]$.

Remark 4.2. The above lemma is a generalization of Propositions 4.1 of [5] and 4.3 of [4], where this result is shown for the special case that $Y$ is the image of the multiple point scheme of a co-rank one finitely determined map germ from $\left(\mathbb{C}^{n}, 0\right)$ to $\left(\mathbb{C}^{n}, 0\right)$ and from $\left(\mathbb{C}^{n}, 0\right)$ to $\left(\mathbb{C}^{p}, 0\right)$ with $n<p$ respectively. We remark that in the cited propositions, the case $k=1$ was not correctly stated. There the number $m_{0}(\Delta(f))$ in the formula is not considered. This mistake was made as a consequence of the set $Z_{k}$ not being described as the schematic union $Z_{k}=f^{-1}\left(P_{k}(Y)\right) \cup \Sigma^{k}(f)$ and only as $Z_{k}=f^{-1}\left(P_{k}(Y)\right)$.

Lemma 4.3. For all $k=0, \ldots, d-1$,

$$
\operatorname{deg}\left(p_{d-k} \circ f \mid Z_{k}\right)=\operatorname{dim}_{\mathbb{C}} \frac{\mathcal{O}_{n}}{\left(I(Z), p_{d-k} \circ f, J\left(p_{d-k+1} \circ f, I(Z)\right)\right)} .
$$

Proof. This follows directly from Remark 2.1.

In order to describe the number $\operatorname{dim}_{\mathbb{C}} \frac{\mathcal{O}_{n}}{\left(I(Z), p_{d-k} \circ f, J\left(p_{d-k+1} \circ f, I(Z)\right)\right)}$ in terms of Milnor numbers of ICIS in $Z$, we introduce the following notation.

Let $p_{d}=\pi_{d}: Y \rightarrow \mathbb{C}^{d}$ be a generic linear projection and for each $k=1, \ldots, d$, let $\pi_{d-k}: \mathbb{C}^{d-k+1} \rightarrow \mathbb{C}^{d-k}$ be a generic linear projection, say $p_{d-k}=\pi_{d-k} \circ \ldots \circ \pi_{d}$. We assume that these projections are sufficiently generic so that they allow us to compute all objects that we use here.

Set

$$
X_{k}=\left(p_{d-k} \circ f\right)^{-1}(0)=V\left(I(Z), p_{d-k} \circ f\right) .
$$

Notice that since $Z$ is a $d$-dimensional ICIS in $\mathbb{C}^{n}, X_{k}$ is a $k$-dimensional ICIS in $\mathbb{C}^{n}$. Furthermore, for any generic linear projection $H: \mathbb{C}^{p} \rightarrow \mathbb{C}$,

$$
X_{k-1}=X_{k} \cap V(H \circ f)=V\left(I(Z), p_{d-k} \circ f, H \circ f\right) .
$$

Define the following invariant related to $f$ : 


\section{Definition 4.4.}

$$
e_{Z}(f)=\operatorname{dim}_{\mathbb{C}} \frac{\mathcal{O}_{n}}{\left(I(Z), J\left(p_{1} \circ f, I(Z)\right)\right)} .
$$

Remark 4.5. If $f:\left(\mathbb{C}^{2}, 0\right) \rightarrow\left(\mathbb{C}^{3}, 0\right)$ is a finitely determined map germ of co-rank one and $Z=D(f)$ is the double point locus of $f$, then $e_{Z}(f)$ coincides with the invariant $e_{D}(f)$ defined by Gaffney in [1, p. 211].

We describe next the polar multiplicities $m_{k}(Y)$, for $k=0, \ldots, d-1$, in terms of Milnor numbers of the ICIS $X_{k}$ defined in the equation (1). We remember that these sets lie in the source and their defining equations are computable; hence their Milnor numbers are also computable.

The following results form a key tool in finding relations among our invariants.

Theorem 4.6 (Lê-Greuel's formula, 7, 3]). Let $X_{1}$ be a germ at zero of an ICIS in $\mathbb{C}^{n}$ and $X$ be a germ at zero of an ICIS defined in $X_{1}$ by $f_{k}=0$. Let $f_{1}, \ldots, f_{k-1}$ be a system of generators of the ideal that defines $X_{1}$ at zero in $\mathbb{C}^{n}$. Then

$$
\mu\left(X_{1}, 0\right)+\mu(X, 0)=\operatorname{dim}_{\mathbb{C}} \frac{\mathcal{O}_{n}}{\left(f_{1}, \ldots, f_{k-1}, J\left(f_{1}, \ldots, f_{k}\right)\right)} .
$$

Remark 4.7. For a zero-dimensional ICIS we use the simpler formula: Let $f$ : $\left(\mathbb{C}^{n}, 0\right) \rightarrow\left(\mathbb{C}^{n}, 0\right)$ be a map germ such that $Z=f^{-1}(0)$ is an ICIS. Then $\mu(Z, 0)=$ $\delta(f)-1$, where $\delta(f)=\operatorname{dim}_{\mathbb{C}} \frac{\mathcal{O}_{n}}{f^{*}\left(m_{n}\right) \mathcal{O}_{n}}$ (see [9, p. 78]).

Now we describe the main result of this section.

Theorem 4.8. Let $f: Z^{d} \rightarrow Y^{d}$ be a finite morphism, where $Z^{d} \subseteq \mathbb{C}^{n}$ and $Y^{d} \subseteq \mathbb{C}^{p}$ are germs of analytic varieties at zero and $Z^{d}$ is an ICIS in $\overline{\mathbb{C}}^{n}$. Then, for the sets $X_{k}$ given in equation (11) we have

(i) if $k=2, \ldots, d-1$, then $m_{k}(Y)=\frac{1}{\operatorname{deg}(f)}\left[\mu\left(X_{k}\right)+\mu\left(X_{k-1}\right)\right]$,

(ii) $m_{1}(Y)=\frac{1}{\operatorname{deg}(f)}\left[\mu\left(X_{1}\right)+\mu\left(X_{0}\right)\right]-m_{0}(\Delta(f))$ and

(iii) $m_{0}(Y)=\frac{1}{\operatorname{deg}(f)}\left[\mu\left(X_{0}\right)+1\right]$.

Proof. Since $\left.X_{k}=\left(p_{d-k} \circ f\right)^{-1}(0)\right)=V\left(I(Z), p_{d-k} \circ f\right)$ is an ICIS, therefore we apply the formula of Theorem 4.6 to obtain for all $k=1, \ldots, d$,

$$
\mu\left(X_{k}\right)+\mu\left(X_{k-1}\right)=\operatorname{dim}_{\mathbb{C}} \frac{\mathcal{O}_{n}}{\left(I(Z), p_{d-k} \circ f, J\left(p_{d-k+1} \circ f, I(Z)\right)\right)} .
$$

Now from Lemma 4.3, for all $k=0, \ldots, d-1$,

$$
\operatorname{dim}_{\mathbb{C}} \frac{\mathcal{O}_{n}}{\left(I(Z), p_{d-k} \circ f, J\left(p_{d-k+1} \circ f, I(Z)\right)\right)}=\operatorname{deg}\left(p_{d-k} \circ f \mid Z_{k}\right) .
$$

Hence $\mu\left(X_{k}\right)+\mu\left(X_{k-1}\right)=\operatorname{deg}\left(p_{d-k} \circ f \mid Z_{k}\right)$ for all $k=1, \ldots, d-1$.

But for $k \neq 1$, we have from Lemma 4.1 that

$$
\operatorname{deg}\left(p_{d-k} \circ f \mid Z_{k}\right)=\operatorname{deg}(f) m_{k}(Y)
$$

and

$$
\operatorname{deg}\left(p_{d-1} \circ f \mid Z_{1}\right)=\operatorname{deg}(f)\left[m_{1}(Y)+m_{0}(\Delta(f))\right] .
$$

Therefore if $k \neq 1, m_{k}(Y)=\frac{1}{\operatorname{deg}(f)}\left[\mu\left(X_{k}\right)+\mu\left(X_{k-1}\right)\right]$ and for $k=1$ we obtain

$$
m_{1}(Y)=\frac{1}{\operatorname{deg}(f)}\left[\mu\left(X_{1}\right)+\mu\left(X_{0}\right)\right]-m_{0}(\Delta(f)),
$$

which proves (i) and (ii). 
Now, since $X_{0}=V\left(p_{d} \circ f, I(Z)\right)$ is a zero-dimensional ICIS in $\mathbb{C}^{n}$ we have from Remark 4.7 that $\mu\left(X_{0}\right)=\delta\left(p_{d} \circ f, I(Z)\right)-1$.

On the other hand, it follows from Remark 2.1 that

$$
\delta\left(p_{d} \circ f, I(Z)\right)=\operatorname{dim}_{\mathbb{C}} \frac{\mathcal{O}_{n}}{\left(I(Z), p_{d} \circ f\right)}=\operatorname{deg}\left(p_{d} \circ f \mid Z\right) .
$$

But as $f$ is finite, $\operatorname{deg}\left(p_{d} \circ f \mid Z\right)=\operatorname{deg}(f) \operatorname{deg}\left(p_{d} \mid f(Z)\right)=\operatorname{deg}(f) m_{0}(Y)$. Therefore

$$
\mu\left(X_{0}\right)=\delta\left(p_{d} \circ f, I(Z)\right)-1=\operatorname{deg}\left(p_{d} \circ f \mid Z\right)-1=\operatorname{deg}(f) m_{0}(Y)-1
$$

and $m_{0}(Y)=\frac{1}{\operatorname{deg}(f)}\left[\mu\left(X_{0}\right)+1\right]$, which proves (iii).

\section{EULER OBSTRUCTION AND POLAR MULTIPLICITIES}

The local Euler obstruction for nonsingular varieties, introduced by MacPherson in 10, in a purely obstructional way, is an invariant that is also associated to the polar invariants. The local Euler obstruction plays an important role in MacPherson's affirmative response to a conjecture of Deligne and Grothendieck on the existence of Chern classes for singular complex algebraic varieties (see [6], 10] and 8 ).

Lê and Teissier in $[8]$ showed a formula to compute the multiplicities of the local polar varieties. With the aid of Gonzalez-Sprinberg's purely algebraic interpretation of the local Euler obstruction given in [2, they showed that the local Euler obstruction is an alternating sum of the multiplicities of the local polar varieties. For a definition and properties of the local Euler obstruction, we refer to [10] and 2. The following result shows how the local Euler obstruction and the polar multiplicities are related.

Theorem 5.1 ([8]). Let $X$ be a germ at zero of a reduced analytic space in $\mathbb{C}^{n}$ of dimension d. Then $E u_{0}(X)=\sum_{k=0}^{d-1}(-1)^{k} m_{k}(X)$, where $m_{k}(X)$ is the $k^{\text {th }}$-polar multiplicity of $X$ at zero and $E u_{0}(X)$ denotes the local Euler obstruction at zero of $X$.

We apply this result to obtain an explicit algebraic formula for the local Euler obstruction of the image of a finite morphism.

Corollary 5.2. Let $f: Z^{d} \rightarrow Y^{d}$ be a finite morphism, where $Z^{d} \subseteq \mathbb{C}^{n}$ and $Y^{d} \subseteq \mathbb{C}^{p}$ are germs at zero of reduced analytic varieties and $Z^{d}$ is an ICIS in $\mathbb{C}^{n}$. Then,

$$
E u_{0}(Y)=\frac{1}{\operatorname{deg}(f)}\left[(-1)^{d} \mu(Z)+1+\operatorname{deg}(f) m_{0}(\Delta(f))+(-1)^{d-1} e_{Z}(f)\right] .
$$

Proof. From Theorem 4.8 we have

(2) $\sum_{i=0}^{d-1}(-1)^{k} m_{k}(Y)=\frac{1}{\operatorname{deg}(f)}\left[(-1)^{d-1} \mu\left(X_{d-1}\right)+1+\operatorname{deg}(f) m_{0}(\Delta(f))\right]$.

Since $\left.X_{d}=\left(p_{0} \circ f\right)^{-1}(0)\right)=V(I(Z))=Z$ is an ICIS, we apply Theorem 4.6 to obtain

$$
\mu\left(X_{d}\right)+\mu\left(X_{d-1}\right)=\operatorname{dim}_{\mathbb{C}} \frac{\mathcal{O}_{n}}{\left(I(Z), J\left(p_{1} \circ f, I(Z)\right)\right)}=e_{Z}(f) .
$$


Hence

$$
(-1)^{d-1} \mu\left(X_{d-1}\right)=(-1)^{d} \mu\left(X_{d}\right)+(-1)^{d-1} e_{Z}(f) .
$$

Therefore, substituting (3) in (2) we get

$$
\sum_{i=0}^{d-1}(-1)^{k} m_{k}(Y)=\frac{1}{\operatorname{deg}(f)}\left[(-1)^{d} \mu(Z)+1+\operatorname{deg}(f) m_{0}(\Delta(f))+(-1)^{d-1} e_{Z}(f)\right],
$$

and the result follows from Theorem 5.1 .

\section{Applications}

In this section we compare our results with some special cases of finite morphisms that recently appeared in the literature.

6.1. Map germs on curves. Nuño-Ballesteros and Tomazella in [13] studied map germs from reduced curves $X$ in $\mathbb{C}^{n}$ to curves $Y$ in $\mathbb{C}^{s}$ and showed how to compute the local polar multiplicity $m_{0}(Y, 0)$.

Theorem 6.1 ([13, Theorem 4.7]). Let $(X, 0)$ be a 1-dimensional ICIS, defined by $(X, 0)=V\left(g_{1}, \ldots, g_{n-1}\right)$ in $\left(\mathbb{C}^{n}, 0\right)$ and $f:(X, 0) \rightarrow\left(\mathbb{C}^{s}, 0\right)$ be a finite function germ onto its image $(Y, 0)$ of degree $\operatorname{deg}(f)$. Then for any generic linear projection $p: \mathbb{C}^{s} \rightarrow \mathbb{C}:$

$$
m_{0}(Y)=\frac{1}{\operatorname{deg}(f)}\left[\operatorname{dim}_{\mathbb{C}} \frac{\mathcal{O}_{n}}{\left(g_{1}, \ldots, g_{n-1}, J\left(g_{1}, \ldots, g_{n-1}, p \circ f\right)\right)}-\mu(X, 0)+1\right] .
$$

Remark 6.2. This result is a special case of Theorem 4.8. We remember that as $Y$ is a curve, its local Euler obstruction at zero coincides with its multiplicity $m_{0}(Y)$.

6.2. Polar multiplicities of multiple points sets. The computation of the polar multiplicities of multiple points sets in the discriminant of a co-rank one finitely determined map germ from $\mathbb{C}^{n}$ to $\mathbb{C}^{p}$ is a special case of Corollary 5.2 .

Theorem 6.3 (4 4 , Theorem 4.4]). Let $f:\left(\mathbb{C}^{n}, 0\right) \rightarrow\left(\mathbb{C}^{p}, 0\right)$ be a finitely determined map germ of co-rank one with $n<p$. Then

$$
\begin{aligned}
\sum_{i=0}^{d-1}(-1)^{i} N(\mathcal{P}) m_{i}( & \left.f\left(D_{1}^{k}(f, \mathcal{P})\right)\right) \\
& =(-1)^{d} \mu\left(D^{k}(f, \mathcal{P})\right)+1+(-1)^{d+1} e_{D_{1}^{k}(f, \mathcal{P})}\left(f_{\mid D_{1}^{k}(f, \mathcal{P})}\right) .
\end{aligned}
$$

In this theorem, $D^{k}(f, \mathcal{P}) \subset \mathbb{C}^{n-1+k}$ denotes the multiple points set of $f$ with respect to a partition $\mathcal{P}$ of $n$. The set $D_{1}^{k}(f, \mathcal{P}) \subset \mathbb{C}^{n}$ is the natural projection of $D^{k}(f, \mathcal{P}) \subset \mathbb{C}^{n-1+k}$ on $\mathbb{C}^{n}$, the number $e_{D_{1}^{k}(f, \mathcal{P})}\left(f_{\mid D_{1}^{k}(f, \mathcal{P})}\right)$ is given in Definition 4.4 and $N(\mathcal{P})$ denotes the degree of $f$ restricted to $D_{1}^{k}(f, \mathcal{P})$. For a precise definition of the scheme structure of these multiple points sets of $f$, we refer to [11].

The case of $n=2$ and $p=3$ was described by Gaffney in [1, Proposition 8.6]. 
For co-rank one map germs from $\left(\mathbb{C}^{n}, 0\right)$ to $\left(\mathbb{C}^{n}, 0\right)$ we have the following:

Theorem 6.4 ([5, Theorem 4.3]). Let $f:\left(\mathbb{C}^{n}, 0\right) \rightarrow\left(\mathbb{C}^{n}, 0\right)$ be a finitely determined map germ of co-rank one. Then,

$$
\begin{aligned}
\sum_{i=0}^{n-m-1}(-1)^{i} N(\mathcal{P}) m_{i}\left(f \circ q\left(D^{\ell}(f, \mathcal{P})\right)\right)=(-1)^{n-m} \mu\left(D^{\ell}(f, \mathcal{P})\right)+1 & \\
& +(-1)^{n-m+1} e_{D^{\ell}(f, \mathcal{P})}\left(f \circ q_{\mid D^{\ell}(f, \mathcal{P})}\right) .
\end{aligned}
$$

In this theorem $D^{\ell}(f, \mathcal{P}) \subset \mathbb{C}^{n-1+\ell}$ denotes the $\ell$-multiple points set of $f, q$ denotes a generic linear projection $q: \mathbb{C}^{n-1+\ell} \rightarrow \mathbb{C}^{n}$ and $N(\mathcal{P})$ denotes the degree of $f \circ q$ restricted to $D^{\ell}(f, \mathcal{P})$.

The case of $n=2$ and $p=2$ was described by Gaffney in [1, Proposition 9.2].

Remark 6.5. These theorems were not correctly stated in the original articles. To correct them we follow the arguments given in Remark 4.2 and add the term $N(\mathcal{P}) m_{0}(\Delta(g))$ in its right-hand side of their equations, where $g=f_{\mid D_{1}^{k}(f, \mathcal{P})}$ in Theorem 6.3 and $g=f \circ q$ in Theorem 6.4 respectively.

\section{REFERENCES}

[1] T. Gaffney, Polar multiplicities and equisingularity of map germs, Topology, 32, No. 1, 185223, 1993. MR1204414 (94f:32072)

[2] G. Gonzalez-Sprinberg, L'obstruction locale d'Euler et le théorème de MacPherson, Astérisque 82 and 83, 7-33, 1978.

[3] E. Brieskorn and G. M. Greuel, Singularities of complete intersection, Manifolds-Tokyo 1973 (Proc. Internat. Conf., Tokyo, 1973), 123-129, Univ. Tokyo Press, Tokyo, 1975. MR 0367273 $(51: 3515)$

[4] V. H. Jorge-Pérez and M. J. Saia, Euler obstruction, polar multiplicities and equisingularity of map germs in $\mathcal{O}(n, p), n<p$. International Journal of Mathematics, 17, 1-17, 2006. MR2261639 (2008a:32025)

[5] V. H. Jorge-Pérez, D. Levcovitz and M. J. Saia, Invariants, equisingularity and Euler obstruction of map germs from $\mathbb{C}^{n}$ to $\mathbb{C}^{n}$. Journal für die reine und angewandte Mathematik, Berlin, 587, 145-167, 2005. MR2186977 (2006k:32054)

[6] G. Kennedy, MacPherson's Chern classes of singular algebraic varieties, Comm. in Algebra 18, 2821-2839, 1990. MR1063344(91h:14010)

[7] D. T. Lê, Calculation of Milnor number of isolated singularity of complete intersection, Funktsional'ny: Analizi Ego Prilozheniya 8, 2, 45-49, 1974. MR0350064 (50:2557)

[8] D. T. Lê and B. Teissier, Variétés polaires locales et classes de Chern des variétés singulières. Annals of Math. (2), 114, 457-491, 1981. MR634426 (83k:32012a)

[9] E. J. N. Looijenga, Isolated singular points on complete intersections, London Mathematical Soc. Lecture Note Series 77, 1984. MR747303 (86a:32021)

[10] R. D. MacPherson, Chern class for singular algebraic varieties, Ann. of Math. (2), 100, 423-432, 1974. MR0361141 (50:13587)

[11] W. L. Marar and D. Mond, Multiple point schemes for co-rank one maps, J. London Math. Soc. 39, 2, 553-567, 1989. MR1002466 (91c:58010)

[12] D. Mumford, Algebraic Geometry I, Projective Varieties. A Series of Comprehensive Studies in Mathematics 221, Springer-Verlag, 1976. MR0453732 (56:11992)

[13] J. J. Nuño-Ballesteros and J. N. Tomazella, Equisingularity of families of map germs on curves (2008), preprint.

[14] B. Teissier, Variétés polaires II: Multiplicités polaires, sections planes, et conditions de Whitney, Actes de la conference de géométrie algébrique à la Rábida, Springer Lecture Notes, 961, 314-491, 1981. MR708342 (85i:32019) 
Departamento de Matemática, UfPB Campus i, Cidade Universitária 58.051-900, João Pessoa, PB, Brazil

E-mail address: roberto@mat.ufpb.br

Departamento de Matemática, ICMC-USP, Caixa Postal 668, 13560-970 São Carlos, SP, BRAZIL

E-mail address: mjsaia@icmc.usp.br

Departamento de Matemática, UfSCar, Caixa Postal 676, 13565-905 São Carlos, SP, BRAZIL

E-mail address: tomazella@dm.ufscar.br 